\title{
Removal of the Temporalis Muscle from Its Origin: Effects on the Size and Shape of the Coronoid Process
}

\author{
T. G. BOYD, W. A. CASTELLI, and D. F. HUELKE \\ Department of Anatomy, University of Michigan, Ann Arbor, Michigan
}

Removal of certain masticatory muscles from their mandibular attachment has produced a decrease in the size of the regions of insertion, particularly of the coronoid process and mandibular angle. ${ }^{1-5}$ In these studies, the temporalis, medial pterygoid, and masseter muscles were removed from their mandibular attachment or were almost totally extirpated. It has been concluded that the maintenance of the form of the bony processes to which these muscles attach is the result of muscle tension. Also, it has been shown that the arteries supplying the coronoid process, angle, and condyle of the rat, guinea pig, monkey, and man arise from vessels that supply the muscles attaching to these processes, and generally not from the inferior alveolar artery which primarily supplies the mandibular body and teeth. ${ }^{6-9}$ Therefore, it would seem that removal of the masticatory muscles from their mandibular attachment would eliminate the arterial supply to these bony processes. If, however, the blood supply of the mandible is maintained while direct muscle tension is decreased or eliminated by separating the masticatory muscles from their cranial attachment, the effect of the lack of muscle tension could be evaluated.

It is the purpose of this study to note the effects on the coronoid process produced by removal of the temporalis muscle from its cranial origin.

\section{Materials and Methods}

Ten guinea pigs were used in this experimental procedure. The animals were anesthetized with sodium pentobarbital ( 32 $\mathrm{mg} . / \mathrm{kg}$. of body weight) administered in-

This investigation was supported in part by USPHS Research Grant DE,-00895 from the National Institute of Dental Research, National Institutes of Health, Bethesda, Md., and Research Grant 1-SO1-FR-05321-01 from the General Research Support Branch, Division of Research Facilities and Resources, Bethesda, Md.

Received for publication May 25, 1966. traperitoneally. Lidocaine $\mathrm{HCl}$ was used as a local field block in the region of surgery. An incision was made over the left ear, through the tela subcutanea and fascia to expose the temporalis muscle, the entire origin of which was removed from the skull. The muscle was then rolled on itself and placed just above the coronoid process. The incision was closed and the guinea pigs were allowed to recover. The right temporalis muscle was left intact to serve as a control. Eighty days after surgery, the guinea pigs were sacrificed, after which the mandibles were removed and cleaned of all soft tissue. Comparison was made between the operated and control sides and between additional controls from guinea pigs of the same age and weight.

\section{Results}

Of the ten surgical specimens, nine mandibles were useful in determining the effects of the surgical procedure; one coronoid process was broken during the removal of the bone. Of the nine specimens, only two showed a definite decrease in size of the coronoid process on the side operated on (Fig. 1, bones $\mathrm{H}$ and $\mathrm{I}$ ). As can be seen, both of these processes were only very small, flattened elevations when compared with those on the side not operated on. In one specimen (bone $\mathrm{G}$ ), there was some flattening of the process, but it was not as noticeable as in the bones $\mathrm{H}$ and $\mathrm{I}$. This process was within the limits of variation in coronoid process height as determined from the reference controls. Six specimens definitely did not show differences in the shape or the size of the right and left coronoid processes.

\section{Discussion}

Most of the experimental procedures designed to show the effects of removal of masticatory muscles on the form of the mandible have indicated that the lack of muscle ten- 

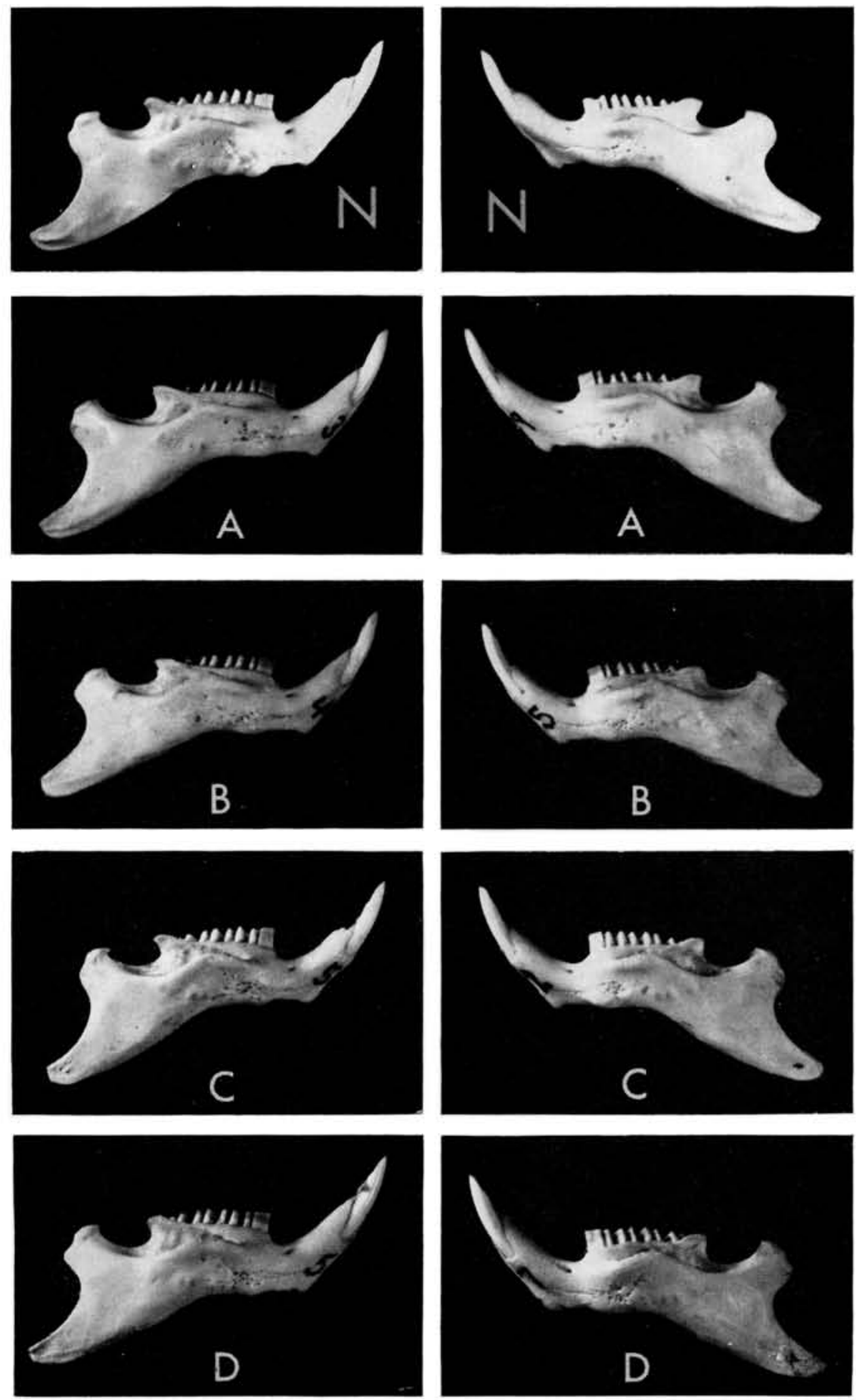

Frg. 1.-Top photographs (N) are from a normal guinea pig that was not operated on. In the other photographs, the side operated on is in the right column, the controls not operated on are on the left. The coronoid processes are the same on both sides of bones 

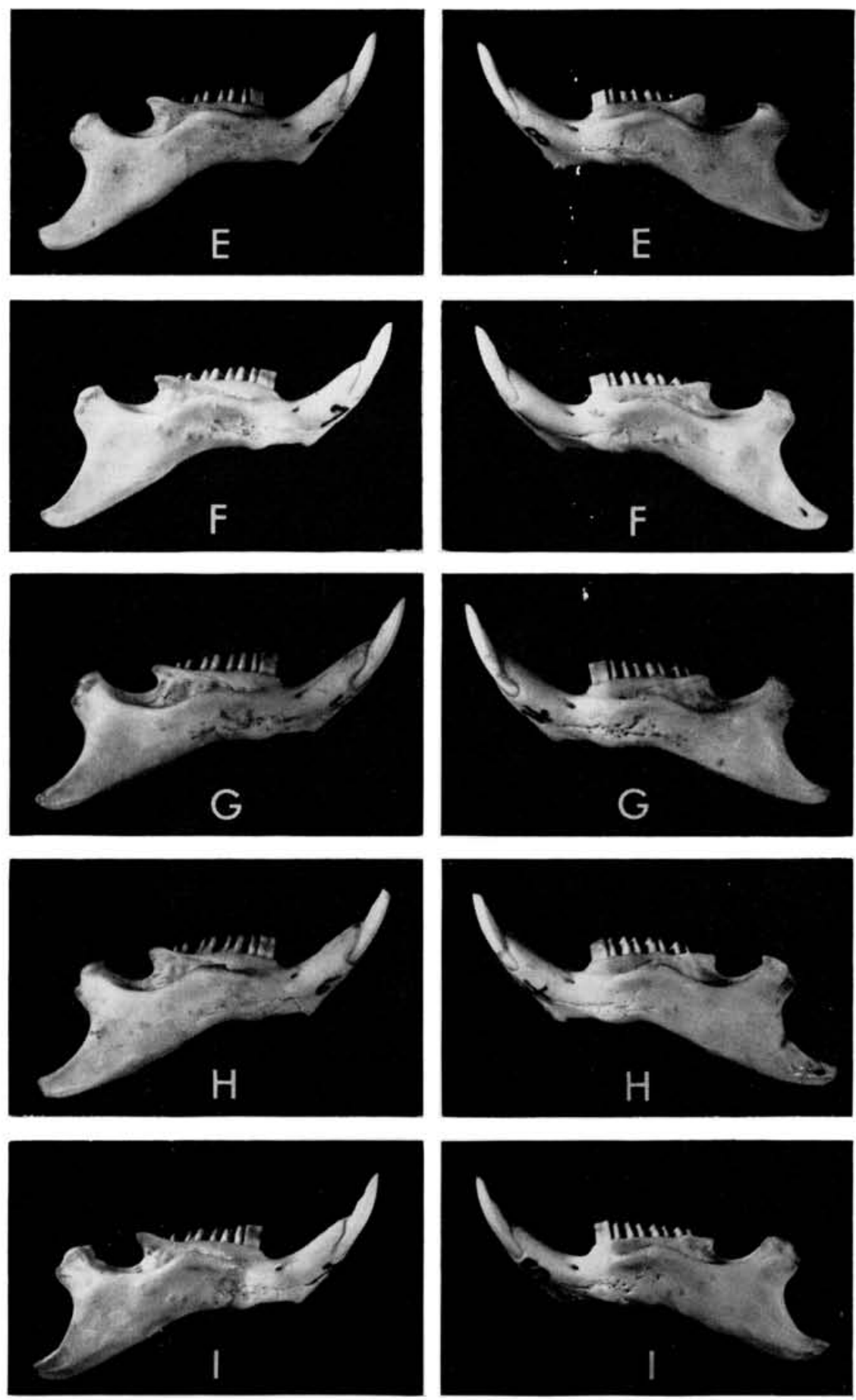

A through F. Bone G showed slight blunting of the coronoid process on the side operated on, but this is within normal limits as indicated by bones B and D (left column). Bones H and $\mathrm{I}$ indicate definite blunting of the process on the side operated on. 
sion produces a decrease in size of the mandible in the region of muscle attachment.

In one of the early experiments, Pratt ${ }^{1}$ removed the masseter muscle on one side in 1-day-old rats. Although no date of sacrifice was indicated, it can be assumed that the rats were adults. He found that there was pronounced asymmetry of both the skull and the mandible and claimed that bone growth seemed definitely related to muscular activity. It can be assumed from his article that there was some loss of bone of the angle, since there was a 9 percent decrease in the angle-coronoid process height. Washburn, ${ }^{2}$ using 1-day-old rats, removed the temporalis muscle from its coronoid process insertion on one side. Three to 5 months later, the coronoid processes on the operated side either were absent or appeared only as small tubercles where a few muscle fibers were yet attached. $\mathrm{He}$ concluded that postnatal growth of the coronoid process is entirely dependent on the presence of a functioning temporalis muscle. Horwitz and Shapiro, ${ }^{3}$ using 8 rats of approximately 30 days of age, removed the temporalis muscle on one side. When the rats were sacrificed, no more than 2 months after the operation, the coronoid process on the operated side was absent. They claimed that "the intrinsic nature of the form of the mandible resulting from the absence of functional stimuli to the part of the mandible providing a site of insertion for the temporalis muscle has been substantiated." These findings are in agreement with those of Washburn. Avis ${ }^{4}$ removed a portion of the temporalis muscle above the zygomatic arch in 6-week-old cats; after 16 months, the coronoid process on the side operated on was somewhat smaller and was vertically oriented instead of being curved backward. Avis claimed that altered mechanical factors produced changes in structure and that the smaller coronoid process resulted from reduced muscle tension. In another study. Avis ${ }^{5}$ removed the superficial masseter muscle, the internal pterygoid muscle, and both of these muscles, in rats 2 to 4 weeks old. She found that 8 to 43 days after the operation the angular process was reduced in all dimensions when only the superficial masseter muscle or the internal pterygoid muscle was removed. The angular process was completely absent in the rats that had had combined operations, where both muscles were removed. If, however, a few fibers of the internal pterygoid or superficial masseter muscle were still present, then a small "atypical" spur of bone was found at the angle. From the results of these experiments, she concluded that the angle is directly related to the functions of the muscle and its attachment to it.

There have been other studies that pertain to this topic. Tower, ${ }^{10}$ using 3 puppies, 6 weeks old, cut all the posterior roots in the lumbosacral region and transected the cord above and below. The dogs lived for 2, 5, and 6 months. The hind bones of the experimental side were about normal in length; however, those regions serving the muscle attachment were deficient. He concluded that the bone thickness and detail are determined by muscle function, but not its length or form. Similarly, Wolffson ${ }^{11}$ removed certain muscles from the scapula either singularly or in groups in rats 1 and 2 days old. After 71 to 82 days, he found that there was a decrease of the bone in the region of the muscle attachment. The brachial plexus was also cut in some animals to demonstrate specifically that it is not the decreased blood supply in the bone which causes the changes in its shape. Wolffson concludes that the shape of the vertebral border and the size of the scapular spine and fossa are greatly affected by muscle function.

In all of the experiments mentioned, only very young animals were used. Certain of the bony markings are obvious at birth-coronoid process and angular process of the mandible - so the question of the cause of regression in these regions when the muscles attached to them are removed can be studied early.

In all instances in which the muscle or muscles were removed from the mandibular angle or coronoid process, the blood supply to these regions was decreased or totally eliminated. Therefore, muscle tension alone cannot explain the decrease in the size of these regions of insertion. This is evident in the statement by Avis ${ }^{5}$ previously cited; she indicated that when a few fibers of the internal pterygoid or masseter muscles were still present, a small "atypical" spur of bone was found at the angle. It seems doubtful that these few muscle fibers still could be functioning if all of the other muscle fibers around it had been removed since the nerve supply undoubtedly had been removed also. It is possible that in this instance the small 
"atypical" spur of bone remained because the bone was receiving nutrient vessels through the few muscle fibers attached to it. This is also true in some of Washburn's ${ }^{2}$ animals in which a spur of a coronoid process remained. Avis'4 discovery of a vertical coronoid process after removal of the origin of the temporalis muscle, was interpreted as the result of altered mechanical factors and changes in the direction of the tension of the temporalis fibers. Although this is probably true, closely associated with it is the fact that the process was maintained because it was still receiving a blood supply through those remaining temporalis fibers arising below the zygomatic arch level which Avis did not remove.

Liebman and Kussick $^{12}$ removed the temporalis muscle from puppies 10 to 14 weeks old. The animals were sacrificed when mature. The only changes were the straightening and vertical alignment of the concaved posterior border of the coronoid process. No shortening of the process was noted. The amount of temporalis muscle removed was not indicated.

In many of the articles mentioned, various authors have contended that the presence of muscle attachment regions, such as the coronoid process, angle, or other tuberosities, result from the muscle tension. Vascularization is important; Felts, ${ }^{13}$ after bone transplantation experiments, concluded that bone growth is dependent on vascularization. Enlow ${ }^{14}$ says that a reevaluation of muscle tension and bone growth concept is needed. His investigations have shown that in vivo muscle pull can be associated with regions undergoing normal cortical recession as well as with cortexes growing in a periosteal direction. Furthermore, he questions whether in vivo muscle pull is directly involved in the control of bone growth. ${ }^{15}$

We are not surprised at the decrease of the coronoid process and mandibular angle in the previous experiments, as well as in the other instances in which the muscles were removed from the scapula, since it is doubtful that the bone will grow without an adequate blood supply. In some specimens, if not in all, there probably was osseous necrosis of the coronoid process. In two or possibly three specimens, a decrease in size of the coronoid process was found. This change could have resulted from interruption of the arterial supply by the surgical proce- dures, or possibly from interference with the nerve supply.

\section{Summary}

Removal of the origin of the temporalis muscle from the skull to decrease or eliminate muscle tension, while still maintaining an intact blood supply, does not cause any changes in the size or shape of the coronoid process in the majority of experimental animals. Removal of the masticatory muscles from the mandible eliminates the blood supply to the regions of insertion and the conclusion drawn from these types of experiments-that muscle tension is needed for maintenance of bony processes-is open to question.

\section{References}

1. Pratt, L. W. Experimental Masseterectomy in the Laboratory Rat, J. Mamm., 24:204-211, 1943.

2. Washburn, S. L. The Relation of the Temporal Muscle to the Form of the Skull, Anat. Rec., 99: $239-248,1947$.

3. Horwitz, S., and Shapiro, H. H. Modifications of Mandibular Architecture Foliowing Removal of the Temporalis Muscle in the Rat., J. Dent. Res., $\mathbf{3 0}: 276-280,1951$

4. Avis, V. The Relation of the Temporal Muscle to the Form of the Coronoid Process, Amer. J. Phys. Anthrop., 17:99-104, 1959.

5. Mandible: An Experimental and Comparative Study, Amer. J. Phys. Anthrop., 19:55-61, 1961.

6. Castelli, W. A. Vasculature Architecture of the Human Adult Mandible, J. Dent. Res., 42:786792,1963 .

7. Castelli, W. A., and Huelke, D. F. The Arterial System of the Head and Neck of the Rhesus Monkey with Emphasis on the External Carotid System, Amer. J. Anat., 116:149-170, 1965.

8. Huelke, D. F., and Castelli, W. A. The Blood Supply of the Rat Mandible, Anat. Rec., 153:335343,1966

9. Boyd, T. G., Castelli, W. A., and Huelke, D. F. Arterial Supply of the Guinea Pig Mandible, J. Dent. Res., 46:1064-67, 1967.

10. Tower, S. S. Tropic Control of Non-Nervous Tissue by the Nervous System: A Study of Muscle and Bone Innervated from an Isolated and Quiescent Region of Spinal Cord, J. Comp. Neurol., 67:241$243 / 257-261,1937$.

11. Wolffson, D. M. Scapula Shape and Muscle Function with Special Reference to the Vertebral Border, Amer. J. Phys. Anthrop., 8:331-341, 1950.

12. Liebman, F. M., and Kussick, L. An Eectromyographic Analysis of Masticatory Muscle Imbalance with Relation to Skeletal Growth in Dogs, J. Dent. Res. 44:768-774, 1965.

13. FELTS, W. J. Transplantation Studies of Factors in Skeletal Organogenesis. I. Subcutaneously Implanted Immature Long Bone of the Rat and Mouse, Amer. J. Phys, Anthrop., 17:201-215, 1957.

14. EnLow, D. F. The Problems of Muscle Tension and the Stimulation of Bone Growth, Anat. Rec., $151: 451.1965$

15. Hoyte, D. A. N., and EnLow, D. H. Wolff's Law and the Problem of Muscle Attachment on Resorptive Surfaces of Bone, Amer. J. Phys. Anthrop., 24:205-213, 1966 . 